\title{
Time evolution of stream interaction region energetic particle spectra in the inner heliosphere
} \author{
W. H. Matthaeus ${ }^{10}$, S. D. Bale ${ }^{11,12}$, and J. C. Kasper ${ }^{13}$ \\ ${ }^{1}$ Department of Astrophysical Sciences, Princeton University, Princeton, NJ 08540, USA \\ e-mail: cjjoyce@princeton.edu \\ ${ }^{2}$ University of New Hampshire, Durham, NH 03824, USA \\ ${ }^{3}$ Goddard Space Flight Center, Greenbelt, MD 20771, USA \\ 4 Jet Propulsion Laboratory, California Institute of Technology, Pasadena, CA 91109, USA \\ 5 Johns Hopkins University Applied Physics Laboratory, Laurel, MD 20723, USA \\ ${ }^{6}$ California Institute of Technology, Pasadena, CA 91125, USA \\ 7 Southwest Research Institute, San Antonio, TX 78228, USA \\ ${ }^{8}$ University of Texas at San Antonio, San Antonio, TX 78249, USA \\ ${ }^{9}$ University of Arizona, Tucson, AZ 85721, USA \\ ${ }^{10}$ University of Delaware, Newark, DE 19716, USA \\ ${ }^{11}$ University of California at Berkeley, Berkeley, CA, USA \\ 12 The Blackett Laboratory, Imperial College London, London, UK \\ ${ }^{13}$ University of Michigan, Ann Arbor, MI, USA
}

C. J. Joyce ${ }^{1}$, D. J. McComas ${ }^{1}$, N. A. Schwadron ${ }^{1,2}$, E. R. Christian ${ }^{3}$, M. E. Wiedenbeck ${ }^{4}$, R. L. McNutt ${ }^{5}$, C. M. S. Cohen ${ }^{6}$, R. A. Leske ${ }^{6}$, R. A. Mewaldt ${ }^{6}$, E. C. Stone ${ }^{6}$, A. W. Labrador ${ }^{6}$, A. J. Davis ${ }^{6}$, A. C. Cummings ${ }^{6}$, D. G. Mitchell ${ }^{5}$, M. E. Hill ${ }^{5}$, E. C. Roelof ${ }^{5}$, R. C. Allen ${ }^{5}$, J. R. Szalay ${ }^{1}$, J. S. Rankin ${ }^{1}$, M. I. Desai ${ }^{7,8}$, J. Giacalone ${ }^{9}$,

Received 2 September 2020 / Accepted 30 March 2021

\begin{abstract}
We analyze an energetic proton event associated with a stream interaction region (SIR) that was observed at Parker Solar Probe on day 320 of 2018 when the spacecraft was just 0.34 AU from the Sun. Using the Integrated Science Investigation of the Sun instrument suite, we perform a spectral analysis of the event and show how the observed spectra evolve over the course of the event. We find that the spectra from the first day of the event are much more consistent with local acceleration at a weak compression, while spectra from later on are more typical of SIR-related events in which particles accelerated at distant shocks dominate. After the first day, the spectra remain approximately constant, which indicates that the modulation of energetic particles during transit from the presumed source region is weaker than previously thought. We argue that these observations can be explained by a sub-Parker spiral magnetic field structure connecting the spacecraft to a source region in the SIR that is relatively close to the Sun. We further propose that acceleration at weak, pre-shock compressions likely plays an important role in observations of SIR-related events in the inner heliosphere and that future modelling of such events should consider acceleration all along the compression region, not just at the distant shock region.
\end{abstract}

Key words. acceleration of particles - solar wind - magnetic fields

\section{Introduction}

Stream interaction regions (SIRs) form at the intersection between fast solar wind streams, which originate from coronal holes, and the slow solar wind. When fast coronal hole streams follow those of the slow solar wind, a compression region forms as the fast solar wind piles up behind the slower one. The SIR continues to corotate with the Sun as it propagates outward, twisting into an Archimedean spiral. The compression strengthens as it propagates outward from the Sun, often steepening into a forward-reverse shock pair a few AU from the Sun (Gosling et al. 1972, 1976).

It is widely believed that these distant shocks are the primary source driving the acceleration of the energetic particle populations associated with SIRs (Barnes \& Simpson 1976; Palmer \& Gosling 1978). The common interpretation of SIRrelated events is that the energetic particles accelerated at these distant shocks propagate back inward toward the Sun, filling the connecting flux tube, and are observed as the corotating compression region passes over the observer, typically located much closer to the Sun than the presumed acceleration region. While distant shocks are commonly thought of as the primary acceleration source, recent studies have shown the importance of the acceleration of suprathermal ions within the SIR compressions at or within $1 \mathrm{AU}$, where shocks are not present (Chotoo et al. 2000; Chen et al. 2015). Observations of these events are generally characterized by energetic particle flux onsets that are more gradual than the events associated with flares or coronal mass ejections (CMEs). Stream interaction regions often show little evidence of energy dispersion or anisotropy, despite their remote source, due to the scattering and mirroring of the sunward propagating energetic particles; however, anisotropies are sometimes observed (Marshall \& Stone 1978). 
ORIGIN, INJECTION, ACCELERATION: OBSERVATIONS

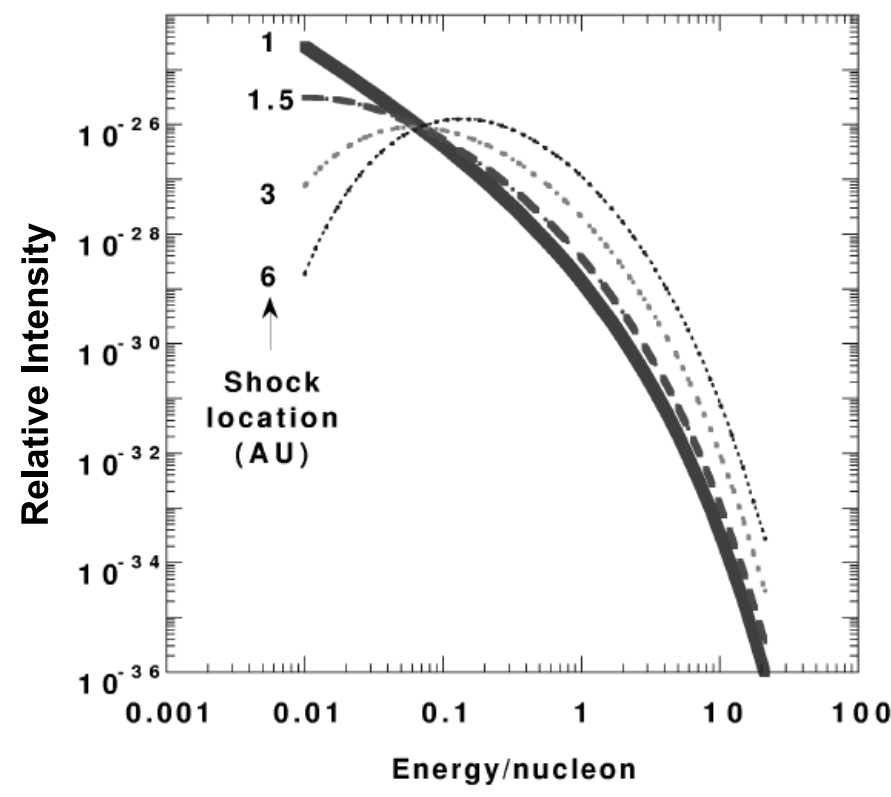

Fig. 1. Figure 6 from Mason et al. (1999), showing energetic particle spectra computed for an observer at $1 \mathrm{AU}$ via the model of Fisk \& Lee (1980) for different distances of the shock region from the Sun.

Fisk \& Lee (1980) provided the traditional model of particle acceleration for energetic particle events associated with SIRs. In this picture, energetic particles are accelerated at the shock region and transported back inward toward the observer. The energy spectrum observed by the spacecraft is thus a product of both the acceleration mechanism and the transport effects acting on the accelerated particles as they stream back inward from the shock. Fisk \& Lee (1980) combine a simple shock model with a computation of the adiabatic deceleration of the energetic particles as they stream back to the observer. Figure 1 shows the energy spectra predicted by this model as a function of distance to the source region. The model predicts that energetic particle observations close to the shock produce a spectrum that steepens somewhat as it transitions from low to high energies. For more distant shocks, the effect of transport on the particles increases and the spectrum softens at high energies while hardening at lower energies, resulting in a more pronounced transition between low and high energies. At sufficiently large distances from the source region, the spectrum rolls over at low energies as those particles are unable to penetrate far enough into the inner heliosphere to reach the observer.

Since the model of Fisk \& Lee (1980) computes spectra for an observer at $1 \mathrm{AU}$, it is not perfectly analogous to the measurements shown here by Parker Solar Probe (PSP) at 0.3 AU. Given the increased magnetic field strengths and distance to the presumed source region, we would expect even stronger transport effects for an observer this close to the Sun. Modeling of the transport of particles from distant shocks to the near-Sun regions explored by PSP is needed for a closer comparison but is beyond the scope of this current work. Nevertheless, it is reasonable to expect that such modeling would produce results qualitatively similar to those of Fisk \& Lee (1980), but with perhaps an even greater depletion of lower energy particles at greater source distances due to the increased strength of the magnetic field closer to the Sun.

The Integrated Science Investigation of the Sun (IS $\odot$ IS) instrument suite (McComas et al. 2016) was launched aboard
PSP on August 12, 2018 (Fox et al. 2016); it is designed to provide a comprehensive analysis of the near-Sun energetic particle environment as PSP repeatedly plunges closer to the Sun than any previous spacecraft. The highly elliptical orbits of PSP, which later in the mission will take it as close as $10 R_{\odot}$ from the Sun, enable IS $\odot$ IS to observe SIR-related energetic particle events over a range of heliocentric distances, yielding new insights into how particle transport affects observations. Early measurements of SIR-related events by IS $\odot$ IS in the inner heliosphere have not shown the low-energy rollover predicted by Fisk \& Lee (1980), despite presumably being well removed from the regions of shock formation (Desai et al. 2020; Joyce et al. 2020). However, spectral indices at lower energies have been significantly harder than those at high energies, indicating distant source regions. This paper analyzes the spectral time evolution of an SIR-related event observed just 0.34 AU from the Sun in an effort to explore how the distance to the SIR acceleration source affects energetic particle observations.

\section{Event overview}

The IS $\odot$ IS instrument suite incorporates two Energetic Particle Instruments, EPI-Hi and EPI-Lo, which are designed to measure energetic particles over higher and lower energy ranges. Combined, IS $\odot$ IS is capable of measuring energetic ion fluxes from $20 \mathrm{keV}$ to $100 \mathrm{MeV}$ per nucleon. Joyce et al. (2020) provided the first analysis of energetic particle events using the combined energy spectrum of EPI-Lo and the first low energy telescope (LET) of EPI-Hi (called LETA), examining seven SIR-related events as well as one impulsive event. We applied a similar method of observation to the analysis of the SIR-related event studied here.

Figure 2 provides an overview of an event characterized by an enhancement in energetic proton fluxes, which began on day of year (DOY) 320 of 2018 and persisted for almost four days. The event has been interpreted as being an SIR event (McComas et al. 2019; Allen et al. 2020; Cohen et al. 2020; Desai et al. 2020; Joyce et al. 2020) related to the compression region that passed over the spacecraft one day prior to the event onset. The event has a gradual onset and decline and shows little evidence of anisotropy or dispersion, which is consistent with most observations of SIR-related events.

McComas et al. (2019) identified the small, very low energy enhancement observed by EPI-Lo at the passage of the SIR at the start of DOY 319 as being evidence of local acceleration at a weak compression. Weaker compressions are capable of accelerating energetic particles even absent a shock under the right conditions via mechanisms similar to diffusive shock acceleration (Giacalone et al. 2002). This fact may be critical in explaining the spectral observations we show in Sect. 3. We note that, while not shown in Fig. 2, a CME passed over the spacecraft on DOY 315 (McComas et al. 2019; Giacalone et al. 2020), approximately five days prior to the observation of the event studied here.

It might be expected that the onset of the energetic particle event associated with the SIR would coincide with the passage of the compression, and it is therefore notable that the event onset is delayed by approximately one day, when the spacecraft was well into the trailing high-stream rarefaction region. This, combined with the four-day duration of the event, suggests that the magnetic field configuration during this time is not the expected Parker spiral orientation but instead must be much more radial in order to connect the spacecraft to the source 
C. J. Joyce et al.: Time evolution of stream interaction region energetic particle spectra in the inner heliosphere

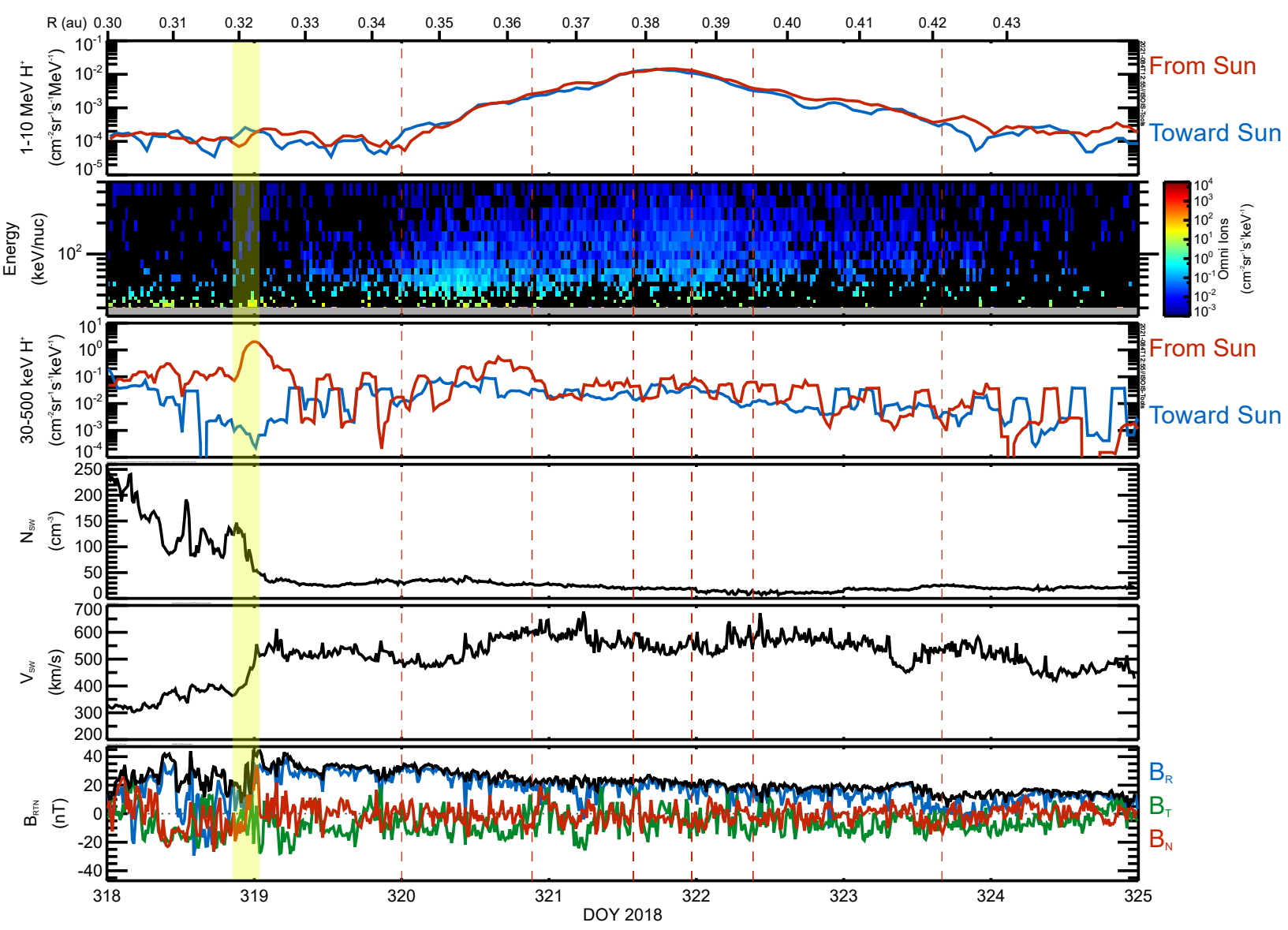

Fig. 2. Overview of the SIR-related event beginning on DOY 320 of 2018. Top three panels: energetic proton enhancements extending for almost four days. Panels 4 and 5: plasma conditions during this period as measured by the Solar Wind Electrons Alphas and Protons (SWEAP) instrument (Kasper et al. 2016); the associated compression region passing over the spacecraft near the beginning of DOY 319 is highlighted in yellow. Bottom panel: magnetic field as measured by the FIELDS instrument (Bale et al. 2016) in RTN (radial, tangential, normal) coordinates (see Fränz \& Harper 2002), showing a highly radial field over this time period. Dotted red lines indicate the divisions between periods used for spectral analysis. The EPI-Lo, plasma, and magnetic field data are averaged over 15-min intervals, while the EPI-Hi data is hourly. No background subtraction has been applied to the data shown here.

region over this period (this is shown in detail by the analysis of Schwadron et al. 2021). Potential implications of this are discussed in Sect. 4. Parker Solar Probe's unique orbits result in very high transverse velocities close to the Sun, and therefore the connection to the source region may have been extended by PSP moving about 10 degrees in the inertial frame during the event in the same sense of rotation as the SIR. As for the SIR, it moved about 58 degrees over the same period, assuming corotation at the mean equatorial sidereal rate of 14.4 degrees/day, typical for solar wind structures (Gosling \& Pizzo 1999).

\section{Spectral analysis}

In order to determine how the spectrum changes over time, we divided the event into five intervals containing equal counts measured by EPI-Lo. Figure 3 shows the proton spectrum measured by IS $\odot$ IS for each interval shown in Fig. 2, as well as the total spectrum for the entire period. Since Fig. 2 shows the event to be relatively isotropic, the spectra are averaged over all look directions to improve statistics. We note that the turnup in the EPI-Lo spectra at higher energies, which is seen particularly in intervals 3 and 5, is due to residual instrument background. A basic background subtraction technique was applied to these observations; however, more sophisticated correction methods are being developed that will improve our ability to resolve the EPI-Lo spectra to higher energies in the future. Details of the spectra computation are provided in the appendix.

Figure 3 shows a significant difference between the observed energetic particle spectrum during the first interval and the rest of the event. The first interval, spanning almost a day, yields a spectrum that is close to a simple power law across the full energy range. After this interval, the spectrum becomes more curved, consistent with acceleration at a more distant source region (as in Fig. 1 ). The overall spectrum for the event is similar to that shown for the same event by Joyce et al. (2020) (referred to as E1 in that work); however, we were able to resolve the spectra to higher energies by averaging over all look directions and incorporating a larger sampling of quiet times for the background spectrum that was scaled and subtracted from the event spectrum.

Except for interval 1, which was fit by a simple power law $\left(j=j_{0} E^{-\gamma}\right)$, each spectrum was fit with a power law with an exponential rollover function of the form $j=j_{0} E^{-\gamma} \exp \left(-E / E_{0}\right)$, with $j$ being the intensity of the spectrum at energy $E, j_{0}$ the normalization constant, $\gamma$ the power-law spectral index, and $E_{0}$ the e-folding energy. The fits were obtained using the same fitting routine as Desai et al. (2020), who used a nonlinear least-squares 

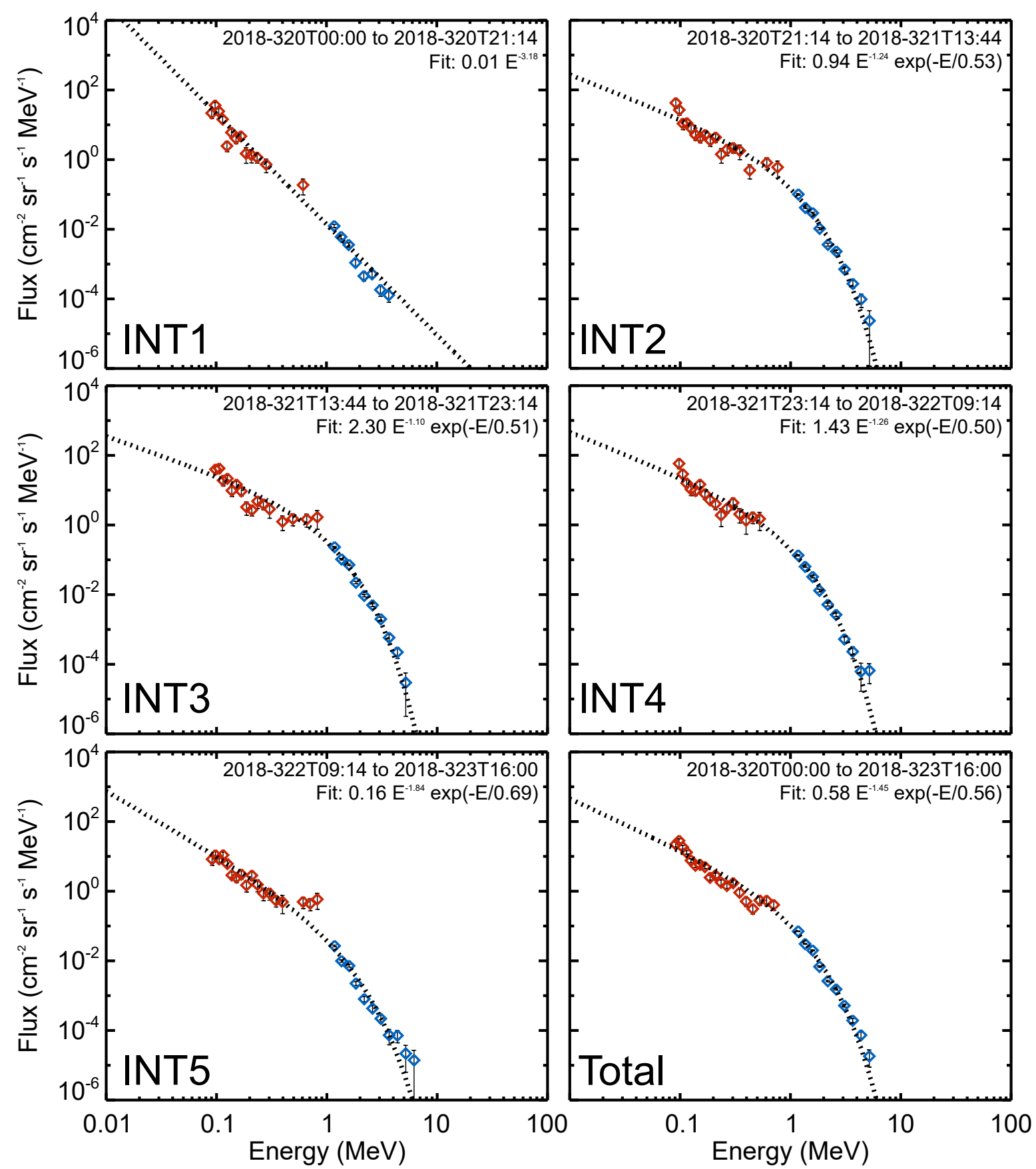

Fig. 3. Proton spectra for five intervals during the SIR-related energetic particle event observed by PSP/IS $\odot I S$ on DOY 320 of 2018 . Red and blue symbols represent low and high energy instruments, respectively, and spectral fits are indicated by dotted black lines. Error bars (often smaller than the symbols) show the statistical uncertainty. To combat the reduced statistics of the triple coincidence EPI-Lo data, re-binning is applied following background subtraction such that each plotted point has at least two counts.

Levenberg-Marquardt technique that minimizes the chi-square value. Fit parameter uncertainties were computed using the offdiagonal terms of the covariance matrix (Markwardt 2009). Fit parameters and uncertainties, as well as reduced $\chi^{2}$ values and probabilities, are given in Table 1.

\section{Discussion}

Table 1 shows the fit parameters for the spectra in Fig. 3. The fits are shown to be quite reasonable, each with probabilities of about $50 \%$. Within the theoretical framework of Fisk \& Lee
(1980), we would expect to see an evolution of the spectrum that is consistent with increasing modulation as the source of the particles becomes more distant later in the event. Instead, we see a spectrum that is quite constant after the first day of the event.

The relative consistency of the spectrum over this period indicates that the modulation of energetic particles during transport is much weaker than predicted by Fisk \& Lee (1980) since the distance to the source region should be increasing during this time, increasing transport effects on the particles. This is notably demonstrated by the relatively constant spectrum of low energy particles during the event (particularly intervals 2, 3, 
Table 1. Fit parameters.

\begin{tabular}{cccccc}
\hline \hline Interval & $j_{0}$ & $\gamma$ & $E_{0}$ & $\chi^{2}$ & Prob \\
\hline 1 & $1.39 \times 10^{-2} \pm 1.80 \times 10^{-3}$ & $3.175 \pm 0.085$ & - & 1.016 & 0.563 \\
2 & $9.36 \times 10^{-1} \pm 2.32 \times 10^{-1}$ & $1.236 \pm 0.147$ & $0.526 \pm 0.051$ & 0.947 & 0.466 \\
3 & $2.30 \times 10^{0} \pm 6.04 \times 10^{-1}$ & $1.097 \pm 0.157$ & $0.511 \pm 0.050$ & 0.967 & 0.497 \\
4 & $1.43 \times 10^{0} \pm 3.59 \times 10^{-1}$ & $1.256 \pm 0.145$ & $0.499 \pm 0.047$ & 0.956 & 0.482 \\
5 & $1.60 \times 10^{-1} \pm 4.69 \times 10^{-2}$ & $1.837 \pm 0.173$ & $0.688 \pm 0.102$ & 0.980 & 0.509 \\
Total & $5.82 \times 10^{-1} \pm 1.35 \times 10^{-1}$ & $1.449 \pm 0.127$ & $0.565 \pm 0.055$ & 1.005 & 0.545 \\
\hline
\end{tabular}

Notes. Each spectrum is fit with a power law with exponential rollover, with the exception of interval 1, which is fit with a simple power law.

and 4), which should be the most affected by modulation due to lower mean free paths (Fisk \& Lee 1980; Mason et al. 1999). When considering this event and others, Desai et al. (2020) proposed that the rarefaction intervals following SIRs may facilitate particle transport due to reduced energy loss from adiabatic deceleration. The apparent lack of modulation also indicates that the magnetic field connecting the spacecraft to the source region deviates significantly from the usual Parker spiral orientation and that particle acceleration is likely occurring not only at a distant shock but over a much broader range of the compression region, extending much closer to the Sun, as we discuss below.

A possible explanation for the connection to a closer source region may be that the local structure of the magnetic field is in a sub-Parker spiral configuration, as described by Schwadron \& McComas (2005), in which the field becomes much more radial than usual (Schwadron et al. 2005; Schwadron 2002). This configuration arises as a result of magnetic foot points moving across the coronal hole boundary, causing the field line to be stretched across the compression region between the fast and slow solar wind streams. This results in a large-scale field orientation that is much less transverse than the nominal Parker spiral (Fig. 2 of Schwadron et al. 2021 provides a diagram) and provides a theoretical framework explaining the radial field configurations that are often observed in the rarefaction regions that follow SIRs (Murphy et al. 2002). The local magnetic field measurements during this period are highly radial, which is consistent with the sub-Parker spiral structure, though the field at this distance from the Sun is expected to be quite radial even for a nominal Parker spiral orientation. In a companion paper, Schwadron et al. (2021) models energetic particle transport to show that the spectral evolution and time-intensity profile shown here can be explained using a sub-Parker spiral magnetic field structure.

This more radial magnetic field configuration may also explain another notable spectral feature of the event: the near single power-law spectrum observed for the first day of the event. Such spectra are often observed during periods where particle acceleration occurs at a localized compression region (Giacalone et al. 2002; Mason 2000). Since the spacecraft was well into the rarefaction region at this point, it is clear that the particles observed during this period were not accelerated locally. However, since the spectrum is consistent with local acceleration and shows little evidence of transport effects acting on the particles, this seems to indicate that the spacecraft is connecting to a portion of the SIR that is relatively close to the Sun, probably before the compression has had time to evolve into a shock. This runs counter to the typical paradigm for SIR-related events that focuses on the acceleration of energetic particles at a shock that forms well beyond $1 \mathrm{AU}$ and their subsequent transport back toward the Sun.
Previous analyses of SIRs have mostly focused on acceleration occurring at distant shocks (McDonald et al. 1976; Marshall \& Stone 1978; Fisk \& Lee 1980; Mason et al. 1999); however, recent studies have shown the significance of acceleration within SIR compressions where shocks are not present (Chotoo et al. 2000; Chen et al. 2015). Based on the observations shown here, we believe that pseudo-localized acceleration at pre-shock compressions may play an important role in observations of SIRrelated events much closer to the Sun. This population may also provide a significant source of seed particles in the inner heliosphere for larger SEP events. As the PSP mission continues, we expect to find many more observations of SIRs close to the Sun and will be able to better determine the importance of acceleration at pre-shock compression regions. For long-duration SIRs, we will be able to make multiple observations of energetic particles associated with the same compression at different distances to the shock source region by combining measurements from PSP and other spacecraft when properly aligned, which should provide greater insight into how transport effects modify energetic particle spectra as a function of distance to the source region.

\section{Summary}

We have provided an analysis of the spectral evolution of an SIRrelated event observed by PSP just 0.34 AU from the Sun. The observations of this event are inconsistent with the usual assumption that energetic particle acceleration for SIR-related events occurs exclusively at distant shocks since we would expect to see more evidence of transport-induced particle modulation in the event spectra. These observations show the potential importance of acceleration at pre-shock compression regions closer to the Sun and that the source region may be considerably larger than previously thought. Understanding the surrounding magnetic field structure during these events is also critical since this determines to which source regions the spacecraft is connected and how far the particles must be transported to reach the observer. A theoretical analysis of how sub-Parker spiral magnetic field structures may explain the characteristics of the event analyzed here is provided in a companion paper by Schwadron et al. (2021). Based on their analysis and the evidence shown here, it seems that this event is best explained by acceleration occurring over a broad source region along the compression combined with a closer connection of PSP to this region as a result of the sub-Parker structure of the magnetic field. Future observations of SIR-related energetic particle events in the inner heliosphere made by PSP, as well as Solar Orbiter, will enable us to determine the importance of these phenomena and improve our understanding of the acceleration and transport of energetic particles associated with SIRs. 
Acknowledgements. This work was supported as a part of the Integrated Science Investigations of the Sun on NASA's Parker Solar Probe mission, under contract NNN06AA01C. The IS $\odot$ IS data and visualization tools are available to the community at: https://spacephysics.princeton.edu/ missions-instruments/isois; data are also available via the NASA Space Physics Data Facility (https://spdf.gsfc.nasa.gov/).

\section{References}

Allen, R. C., Lario, D., Odstrcil, D., et al. 2020, ApJS, 246, 36

Bale, S. D., Goetz, K., Harvey, P. R., et al. 2016, Space Sci. Rev., 204, 49

Barnes, C. W., \& Simpson, J. A. 1976, ApJ, 210, L91

Chen, J. H., Schwadron, N. A., Möbius, E., \& Gorby, M. 2015, J. Geophys. Res Space Phys., 120, 9269

Chotoo, K., Schwadron, N. A., Mason, G. M., et al. 2000, J. Geophys. Res., 105, 23107

Cohen, C. M. S., Christian, E. R., Cummings, A. C., et al. 2020, ApJS, 246, 20

Desai, M. I., Mitchell, D. G., Szalay, J. R., et al. 2020, ApJS, 246, 56

Fisk, L. A., \& Lee, M. A. 1980, ApJ, 237, 620

Fox, N. J., Velli, M. C., Bale, S. D., et al. 2016, Space Sci. Rev., 204, 7

Fränz, M., \& Harper, D. 2002, Planet. Space Sci., 50, 217

Giacalone, J., Jokipii, J. R., \& Kóta, J. 2002, ApJ, 573, 845

Giacalone, J., Mitchell, D. G., Allen, R. C., et al. 2020, ApJS, 246, 29

Gosling, J. T., \& Pizzo, V. J. 1999, Space Sci. Rev., 89, 21

Gosling, J. T., Hundhausen, A. J., Pizzo, V., \& Asbridge, J. R. 1972 J. Geophys. Res., 77, 5442

Gosling, J. T., Hundhausen, A. J., \& Bame, S. J. 1976, J. Geophys. Res., 81, 211
Hill, M. E., Mitchell, D. G., Andrews, G. B., et al. 2017, J. Geophys. Res. Space Phys., 122, 1513

Hill, M. E., Mitchell, D. G., Allen, R. C., et al. 2020, ApJS, 246, 65

Joyce, C. J., McComas, D. J., Christian, E. R., et al. 2020, ApJS, 246, 4

Kasper, J. C., Abiad, R., Austin, G., et al. 2016, Space Sci. Rev., 204, 131

Kecskemety, K., Mueller-Mellin, R., \& Kunow, H. 2001, Int. Cosmic Ray Conf., 8, 3108

Logachev, Y. I., Kecskeméty, K., \& Zeldovich, M. A. 2002, Sol. Phys., 208, 141

Markwardt, C. B. 2009, ASP Conf. Ser., 411, 251

Marshall, F. E., \& Stone, E. C. 1978, J. Geophys. Res., 83, 3289

Mason, G. M. 2000, ASP Conf. Ser., 528, 234

Mason, G. M., Von Steiger, R., Decker, R. B., et al. 1999, in Corotating Interaction Regions, eds. A. Balogh, J. T. Gosling, J. R. Jokipii, R. Kallenbach, \& H. Kunow (Dordrecht: Springer Netherlands), 327

McComas, D. J., Alexander, N., Angold, N., et al. 2016, Space Sci. Rev., 204 187

McComas, D. J., Christian, E. R., Cohen, C. M. S., et al. 2019, Nature, 576, 223 McDonald, F. B., Teegarden, B. J., Trainor, J. H., von Rosenvinge, T. T., \& Webber, W. R. 1976, ApJ, 203, L149

Murphy, N., Smith, E. J., \& Schwadron, N. A. 2002, Geophys. Res. Lett., 29, 23 Palmer, I. D., \& Gosling, J. T. 1978, J. Geophys. Res., 83, 2037

Schwadron, N. A. 2002, Geophys. Res. Lett., 29, 8

Schwadron, N. A., \& McComas, D. J. 2005, Geophys. Res. Lett., 32

Schwadron, N. A., McComas, D. J., Elliott, H. A., et al. 2005, J. Geophys. Res. Space Phys., 110, A04104

Schwadron, N. A., Joyce, C. J., Aly, A., et al. 2021, A\&A

Wiedenbeck, M. E., Angold, N. G., Birdwell, B., et al. 2017, Int. Cosmic Ray Conf., 301, 16 


\section{Appendix A: Details on spectral computation and background subtraction}

Table A.1. Quiet times used to compute the overall background spectrum that is scaled and subtracted from the event spectra in this analysis.

\begin{tabular}{|c|c|}
\hline Start time & End time \\
\hline 2018-296T14:00:00 & 2018-314T16:00:00 \\
\hline 2018-325T08:00:00 & 2018-327T00:00:00 \\
\hline 2019-007T00:00:00 & 2019-011T12:00:00 \\
\hline 2019-023T00:00:00 & 2019-026T15:00:00 \\
\hline 2019-041 T08:00:00 & 2019-044T18:00:00 \\
\hline 2019-052T02:00:00 & 2019-054T12:00:00 \\
\hline 2019-056T00:00:00 & 2019-057T10:00:00 \\
\hline 2019-068Т07:00:00 & 2019-073T00:00:00 \\
\hline 2019-074T12:00:00 & 2019-076T12:00:00 \\
\hline 2019-085T02:00:00 & 2019-092T06:00:00 \\
\hline 2019-139T02:00:00 & 2019-143Т06:00:00 \\
\hline 2019-152Т06:00:00 & 2019-167T10:00:00 \\
\hline 2019-181T09:00:00 & 2019-191T18:00:00 \\
\hline 2019-235T05:00:00 & 2019-237T10:00:00 \\
\hline 2019-248T01:00:00 & 2019-252T17:00:00 \\
\hline 2019-254T08:00:00 & 2019-256T21:00:00 \\
\hline 2019-257T12:00:00 & 2019-259T00:00:00 \\
\hline 2019-260T09:00:00 & 2019-261 T17:00:00 \\
\hline 2019-266T00:00:00 & 2019-268T00:00:00 \\
\hline 2019-278T00:00:00 & 2019-286T00:00:00 \\
\hline 2019-291T00:00:00 & 2019-294T00:00:00 \\
\hline 2019-305T00:00:00 & 2019-316T12:00:00 \\
\hline 2019-345T00:00:00 & 2019-350T00:00:00 \\
\hline 2019-360T00:00:00 & 2020-018T00:00:00 \\
\hline 2020-037T00:00:00 & 2020-046T00:00:00 \\
\hline 2020-053Т00:00:00 & 2020-086T00:00:00 \\
\hline 2020-088T00:00:00 & 2020-105T00:00:00 \\
\hline 2020-109T00:00:00 & 2020-136T00:00:00 \\
\hline 2020-138T00:00:00 & 2020-142T00:00:00 \\
\hline 2020-171T00:00:00 & 2020-182T00:00:00 \\
\hline
\end{tabular}

EPI-Hi (Wiedenbeck et al. 2017) measures ions from 1 to 100 MeV nuc ${ }^{-1}$ using five telescopes of stacked solid-state detectors (SSDs). This includes three LETs and two high energy telescopes (HETs). Both the HETs and LETs have one telescope facing sunward along the nominal Parker spiral and one facing in the reverse direction, with the third LET being perpendicular to the other two in the ram-facing direction (for nominal spacecraft pointing). Particles are characterized by the energy deposited in each SSD as they propagate through the telescope. The sources of background for EPI-Hi in this study are cosmic rays and solar-heliospheric ( $\mathrm{SH})$ particles. Cosmic rays are high energy particles that originate from outside the heliosphere and are expected to increase in intensity with heliocentric distance as a result of decreasing modulation by the interplanetary magnetic field. Solar-heliospheric particles are 1-10 MeV particles of solar origin that are persistently present in the inner heliosphere (Logachev et al. 2002) and decrease in intensity with solar distance (Kecskemety et al. 2001). These sources of background differ from those of EPI-Lo since they constitute accurate measurements of particles that EPI-Hi is designed to measure; however, they are treated as background in this analysis since we focus on the energetic particle population produced by the SIR studied here. The EPI-Hi flux spectra shown in this analysis are averaged across the three LETs (LETA, LETB, and LETC) to provide a wide angular coverage and improve statistics.

EPI-Lo (Hill et al. 2017) is a time of flight (TOF) mass spectrometer with 80 apertures, which cover half of the sky; it is capable of measuring ions from $20 \mathrm{keV}$ to $10 \mathrm{MeV}$ per nucleon. The spectra computed in this study are omnidirectional but exclude look directions $25,31,34,35$, and 44 due to higher levels of background from photon contamination in those apertures. This analysis computes spectra, computing triple coincidence data that combine the TOF measurements with the coincident measurements of energy deposited in SSDs. This enables the determination of species by onboard processing and greatly reduces background from cross-talk and accidentals, which generate false measurements. The primary sources of background for EPI-Lo are ultraviolet photons, which can trigger starts or stops in the instrument, and cosmic rays, which can trigger starts or stops, can generate cross-talk between the detectors, and can be measured by the SSDs. Most cross-talk and accidentals are filtered out by the logic of the instrument's onboard processing, particularly for triple-coincidence data; however, a small fraction of these measurements make their way into the data and must be accounted for during analysis. A more detailed discussion of the backgrounds that affect the EPI-Lo measurements is provided by Hill et al. (2020).

In order to properly characterize the spectra of the event studied here, it is desirable to remove these sources of background from the observations. Analysis of the spectra taken during quiet times over the course of the PSP mission have shown a consistent form with small statistical variations. The main variability of these spectra over time is the overall magnitude, which changes somewhat over time based on various conditions, although a clear pattern is not yet evident (Joyce et al. 2020). We therefore applied the background subtraction technique of Joyce et al. (2020), in which the aggregated spectrum from all quiet times observed thus far by IS $\odot$ IS is scaled by the total flux of a quiet period close to the event and subtracted from the event spectrum. For this analysis, the reference quiet time used for scaling the background is from 2018-311T00:00:00 to 2018-314T16:00:00, and the full set of quiet times used to create the overall background spectrum is listed in Table A.1. 